EPJ Web of Conferences 43, 05007 (2013)

DOI: $10.1051 /$ epjconf/20134305007

(C) Owned by the authors, published by EDP Sciences, 2013

\title{
Quantitative asteroseismology: Determination of the core composition and layering of the ZZ Ceti star R548. Part I
}

\author{
N. Giammichele ${ }^{a}$, G. Fontaine and P. Brassard \\ Département de Physique, Université de Montréal, CP. 6128, Succursale Centre-Ville, \\ Montréal, QC H3C 3J7, Canada
}

\begin{abstract}
We present a detailed asteroseismological study of the pulsating white dwarf R548 based on fits to newly detected periods and the use of the forward method. In this first part, we concentrate on the frequency extraction analysis. Based on an unexploited CFHT/LAPOUNE broadband photometric campaign of high $\mathrm{S} / \mathrm{N}$, we are able to obtain a finer frequency spectrum and uncover two triplets, two doublets, and two singlets. The low-amplitude and "simple" pulsator R548 is an ideal candidate for carrying on a complete asteroseismological analysis within the framework of the adiabatic approximation to obtain optimized structural parameters, including the envelope layering and the bulk composition of the core.
\end{abstract}

\section{INTRODUCTION}

Asteroseismology is a unique tool that allows to probe the internal structure and the evolutionary state of a pulsating star. By comparing the pulsation properties with those predicted by refined theoretical models of stars, it is possible to independently unveil unique features of the stars such as the onion-like chemical structure in pulsating white dwarfs, for example. This method has been applied and slowly perfected over the past two decades on the study of many compact pulsators, and in particular very successfully realised upon the class of hot pulsating B subdwarf stars (see, e.g., the review of [1] for more details).

Despite valiant attempts (see [2] for a historical account), reliable and convincing seismic models of pulsating white dwarfs, particularly those with an hydrogen dominated atmosphere, the ZZ Ceti stars, have remained far and few. Unfortunately enough, cool ZZ Ceti stars tend to show rather complicated pulsation spectra composed of numerous harmonics, frequency sum and difference peaks due to strongly nonlinear pulse features. Their mode identification can even be further complicated by dramatic variations of the pulsation spectra with time. Without the exact pulsation spectrum, it is impossible to pinpoint the corresponding internal structure as the resulting theoretical spectrum is very sensitive to any changes in the parameters of the model. On the other hand, ZZ Ceti variables closer to the blue edge tend to present too few pulsation frequencies, limiting greatly any possible analysis due to the degeneracy of the possible solutions in parameter space. This is the main reason for focusing our efforts on pulsators closer to the blue edge of the instability strip, with the condition of having a reasonable minimal number of pulsation modes.

$\mathrm{R} 548$, the prototype star of the ZZ Ceti class, is one of the low-amplitude pulsator found in the hotter region of the instability strip, showing a quite stable and rather uncomplicated light curve with several independent modes. While it is also one of the most well observed ZZ Ceti star, the few asteroseismological studies realised so far gave mitigated results. Best fits using evolutionnary models

\footnotetext{
a e-mail: noemi@astro.umontreal.ca
}

This is an Open Access article distributed under the terms of the Creative Commons Attribution License 2.0, which permits unrestricted use, distribution, and reproduction in any medium, provided the original work is properly cited. 


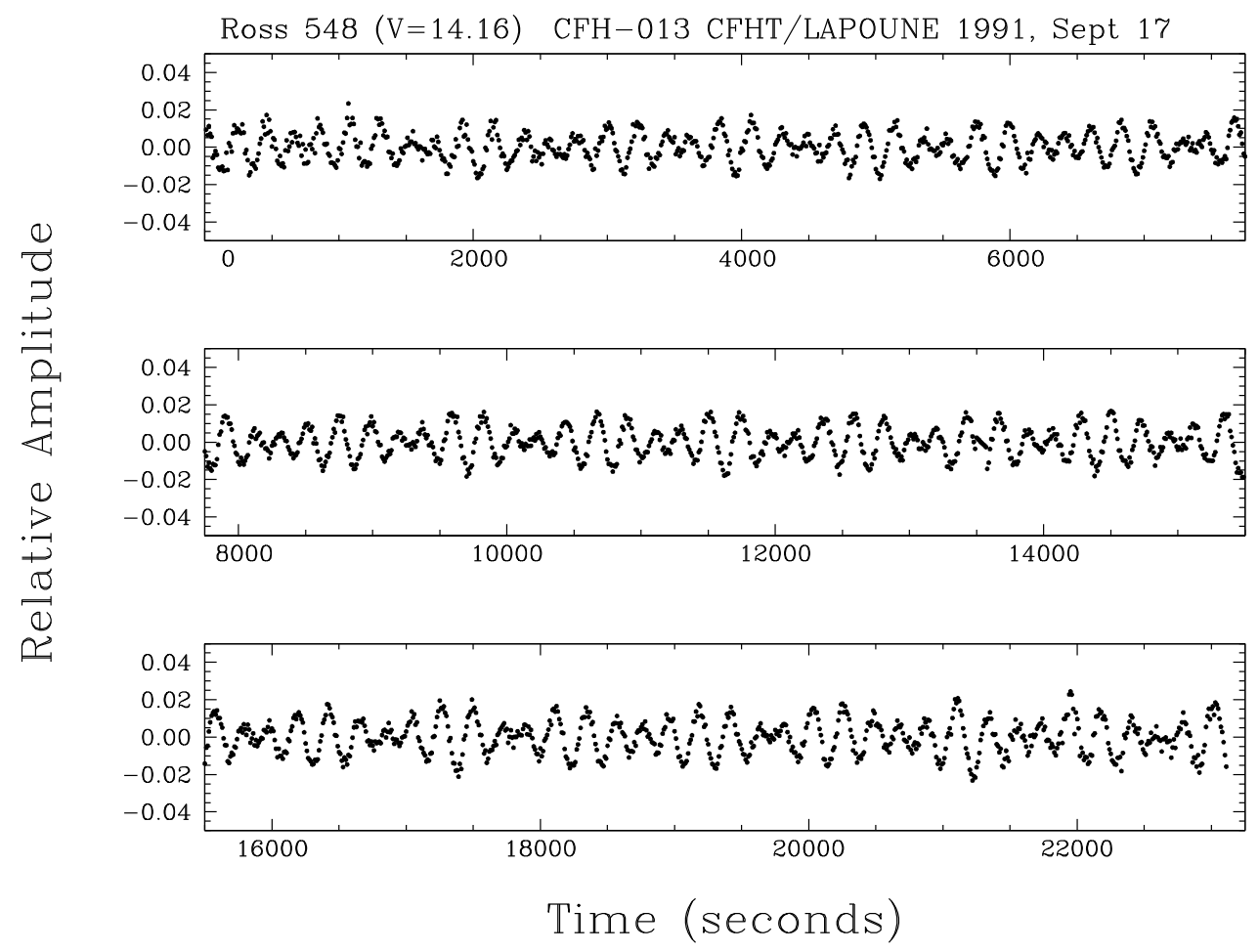

Figure 1. Representative broadband optical light curve obtained in one night for R548. Other nights are similar in length and quality. Each plotted point represents a time sampling of $10 \mathrm{~s}$. The amplitude is expressed in term of residual intensity relative to the mean brightness of the star.

found by [3] and [4], do not seem to precisely narrow down the thickness of the hydrogen layer and give non-negligeable discrepancies between the observed and theoretical period spectrum, of the order of a few seconds per mode.

We have developed at Université de Montréal an approach for quantitative seismic analyses of compact pulsators based on large scale computations relying on a cluster of dedicated computers and the use of parametrized static models. The latter allow us the needed flexibility to explore in great details parameter space, a task that cannot be performed with evolutionary models. We apply this approach to R548 in the present work, a star for which a very high quality photometric data set has been obtained at the CFHT in the past, but has remained largely unexploited. In this report, we present the results of a frequency extraction analysis that we carried out on this data set. The subsequent search for a suitable seismic model in parameter space will be presented elsewhere in the second and final part of our work ([5]).

\section{LIGHT CURVE PROCESSING}

The photometric data processed in this study was taken from the campaign carried out in September 1991, using the CFHT run with the LAPOUNE instrument, a 3-channel portable photometer. The broadband "white light" photometric data extracted was sampled for a total of $19 \mathrm{~h}$ over four nights. Figure 1 shows a typical light curve obtained for one of the observing night. Each plotted point represents a sampling time of $10 \mathrm{~s}$, whereas the amplitude is expressed relatively to the mean brightness of the star. As can be seen, the light curve expectedly shows a rather low-amplitude variation. 
Ageing Low Mass Stars: From Red Giants to White Dwarfs

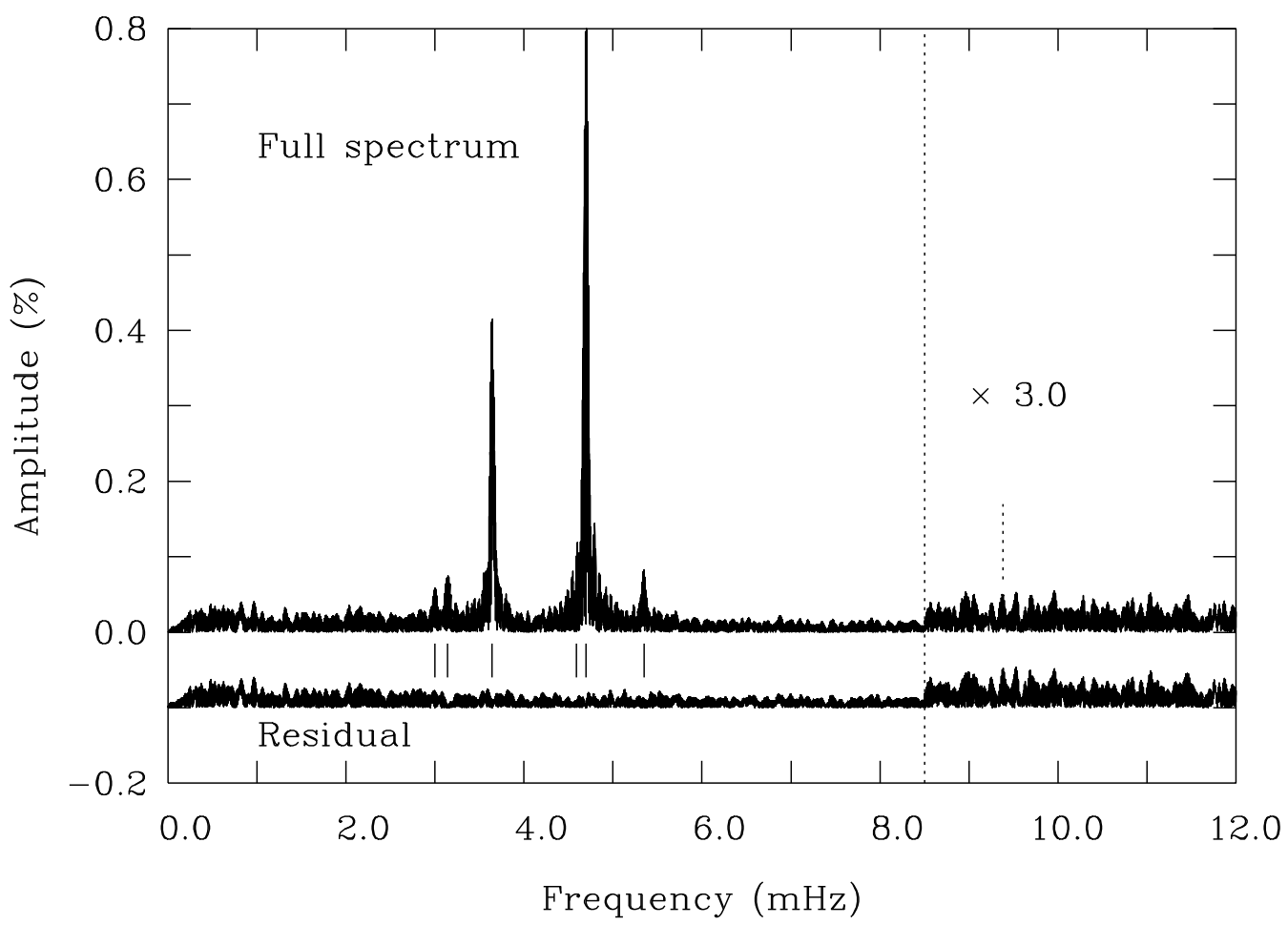

Figure 2. Fourier amplitude spectrum of the R548 light curve. A total of 12 independent periodicities were uncovered out of 6 main peaks, which include 2 triplets, 2 doublets, and 2 singlets. The only nonlinear frequency peak observed — the first harmonic of the dominant peak — is identified and magnified on the right.

The frequency resolution obtained during this run was $2.76 \mu \mathrm{Hz}$ and the duty cycle of $18.8 \%$. Despite the low value of the duty cycle, the signal-to-noise ratio of the obtained spectrum is quite remarkable, one of the best ever obtained on a ZZ Ceti star.

Standard Fourier transform techniques followed by least-square fits and prewhitening sequences were performed on the light curve. The resulting Fourier spectrum and its residual after extracting all apparently significant signals are illustrated in Fig. 2. Out of the six main frequency peaks, we uncovered a total of 12 modes, including two triplets, two doublets, and two singlets. We can notice though that the Fourier spectrum is mainly dominated by the 2 triplets complexes at $213 \mathrm{~s}$ and $274 \mathrm{~s}$. The only nonlinear feature of the light curve of R548, the first harmonic of the dominant mode of the main complex at 213 $\mathrm{s}$ is magnified on the right part of Fig. 2. The residual intensity from the prewhitened curve is shown at the bottom of figure. It is the first check that every feature above the noise level was correctly identified in our analysis.

Table 1 summarizes the details of the extracted periodicities : the periods $P$, amplitudes $A$, frequencies $v$, and the frequency spacing inside a complex $\Delta v$. Uncertainties on the period, amplitude and frequency are drawn from the formalism proposed by [6]. Except for the $217.8362 \mathrm{~s}$ mode and the 318.7672 period, the $\mathrm{S} / \mathrm{N}$ reached in amplitude is above $5 \sigma$.

Figure 3 shows the prewhitening sequences for each of the six main frequency peaks detected in the Fourier transform of the CFHT light curve on R548. The segments of the Fourier transform are vertically and horizontally shifted for a better vizualization. Each column illustrates from top to bottom, the prewhitening sequence obtained for a given peak indentified by its approximate central period (in s). Here we can distinguish the two triplets, two doublets, and the two singlets presented before. It is likely 
Table 1. Observed pulsation properties of R548.

\begin{tabular}{cccccc}
\hline$P(\mathrm{~s})$ & $A(\%)$ & $\mathrm{S} / \mathrm{N}$ & $\nu(\mu \mathrm{Hz})$ & $\Delta \nu(\mu \mathrm{Hz})$ & \\
\hline $186.4882(0.0068)$ & $0.0411(0.0053)$ & 5.8 & $5362.27(0.196)$ & 22.618 & $f_{1}$ \\
$187.2781(0.0047)$ & $0.0597(0.0053)$ & 9.4 & $5339.65(0.135)$ & & $\ldots$ \\
& & & & & \\
$212.7684(0.0009)$ & $0.4201(0.0056)$ & 59.7 & $4699.94(0.020)$ & 3.972 & $\ldots$ \\
$212.9484(0.0028)$ & $0.1394(0.0056)$ & 19.7 & $4695.97(0.062)$ & $<4.015>$ & $f_{2}$ \\
$213.1326(0.0006)$ & $0.6658(0.0056)$ & 95.1 & $4691.91(0.013)$ & 4.058 & $\ldots$ \\
& & & & & \\
$217.8362(0.0131)$ & $0.0312(0.0057)$ & 4.5 & $4590.61(0.275)$ & & $f_{3}$ \\
& & & & & \\
$274.2508(0.0017)$ & $0.4346(0.0063)$ & 55.0 & $3646.30(0.022)$ & 3.647 & $\ldots$ \\
$274.5254(0.0063)$ & $0.1144(0.0063)$ & 14.7 & $3642.65(0.084)$ & $<3.475>$ & $f_{4}$ \\
$274.7745(0.0023)$ & $0.3140(0.0063)$ & 39.8 & $3639.35(0.030)$ & 3.302 & $\ldots$ \\
& & & & & \\
$318.0671(0.0144)$ & $0.0734(0.0069)$ & 8.4 & $3143.99(0.142)$ & 6.905 & $f_{5}$ \\
$318.7672(0.0356)$ & $0.0299(0.0069)$ & 3.6 & $3137.09(0.350)$ & & $\ldots$ \\
$333.6421(0.0196)$ & $0.0622(0.0072)$ & 6.9 & $2997.22(0.176)$ & & $f_{6}$ \\
\hline
\end{tabular}

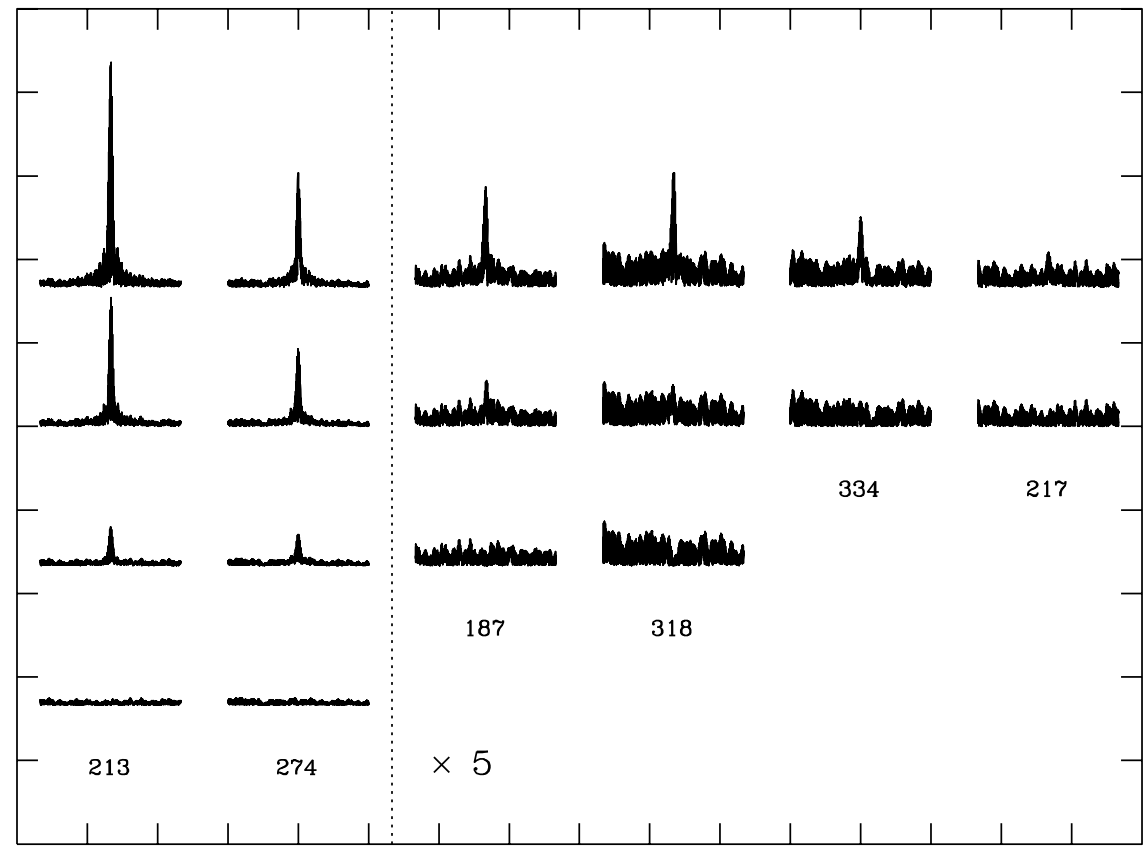

Figure 3. Prewhitening sequences of the R548 light curve. The various prewhitened sequences, are shifted vertically for better clarity (from top to bottom) and are identified by their central period (s). One tick mark in abscissa corresponds to $1 \mathrm{mHz}$, and one in ordinate to $0.2 \%$.

that there are multiplet components in the doublet and the singlet features that have not been detected because their amplitude are smaller than the detection level.

Figure 4 and Fig. 5 provide a zoomed-in view of the two triplet complexes. The reconstructed Fourier transform is superimposed on the actual "observed" Fourier transform at the top of the figure. 
Ageing Low Mass Stars: From Red Giants to White Dwarfs
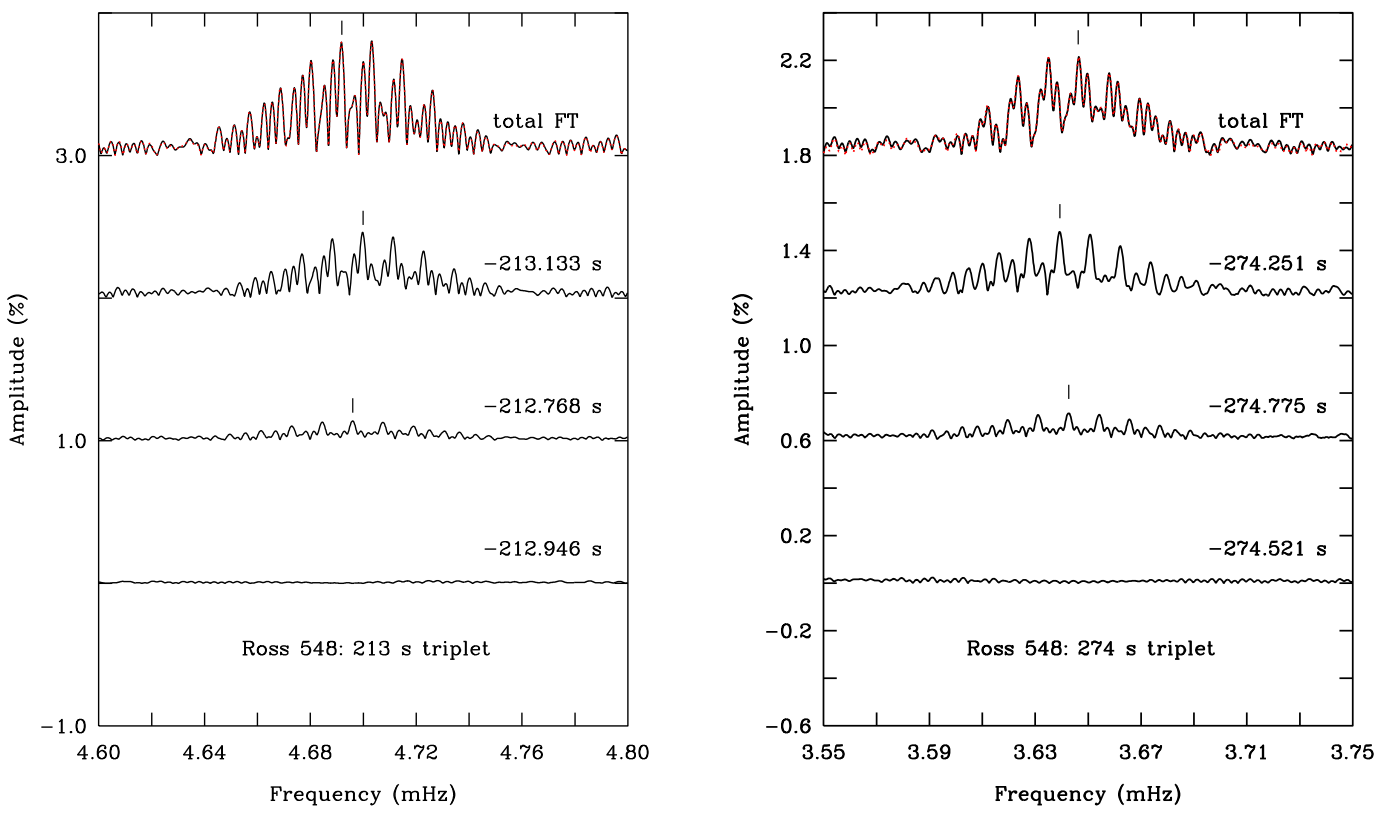

Figure 4. Prewhitening sequence for the $213 \mathrm{~s}$ feature. Figure 5. Prewhitening sequence for the $274 \mathrm{~s}$ feature.

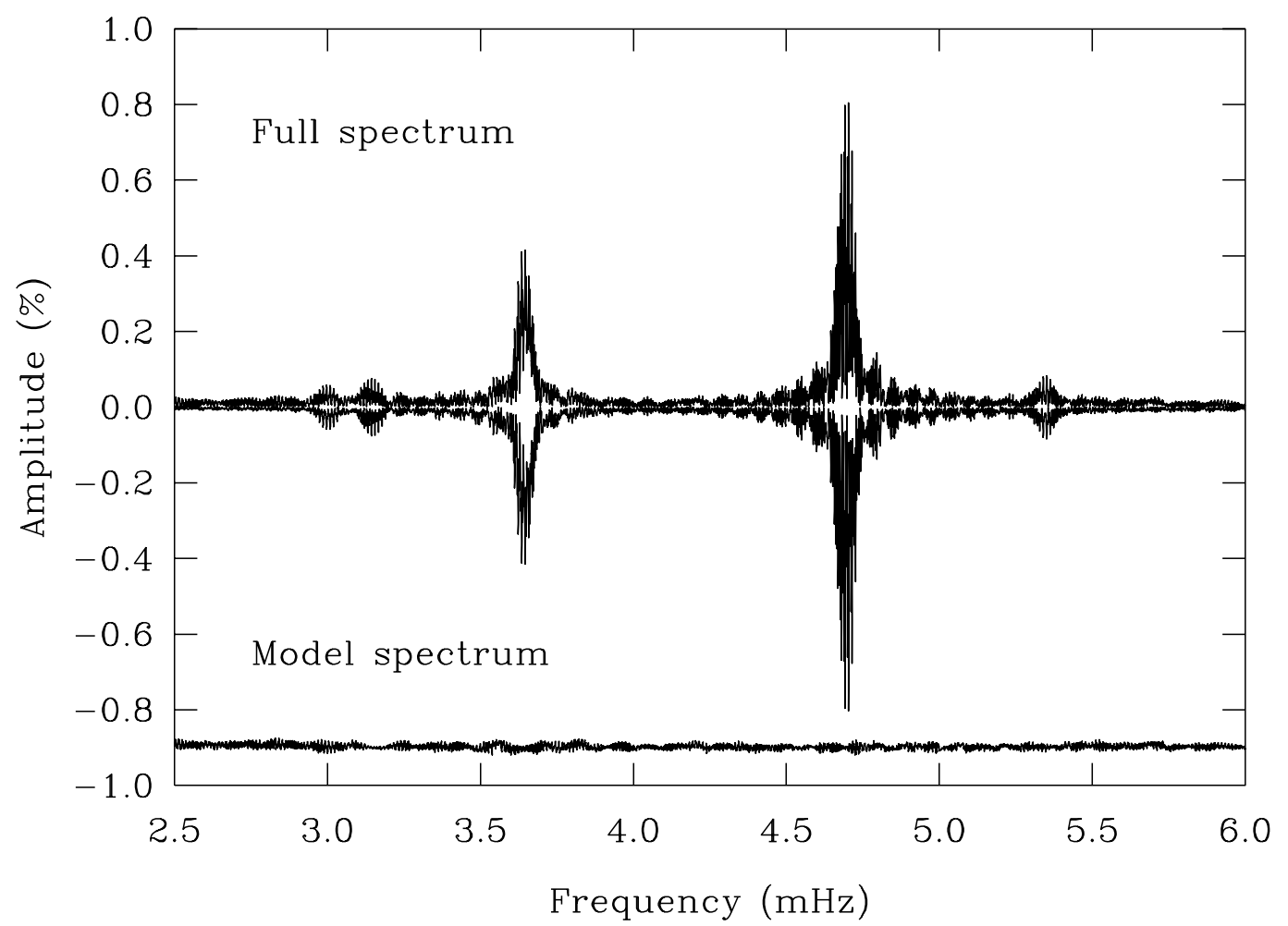

Figure 6. Modeled and observed Fourier transform. The point-by-point subtraction from the two curves is also shown at the bottom of the figure. 
The absence of any noticeable differences between the reconstructed and the "observed" Fourier transforms emphasizes the righteous choice of extracted periods within the two multiplets. The resulting noise level after the two complete prewhitening sequences is very low, which is also a good indication that the extracted frequencies are appropriate. The observed spacings between the frequency components within these multiplets most likely indicate rotational splitting due to the slow rotation of the star. We note that, for over more than two decades, the central components of the $213 \mathrm{~s}$ and $274 \mathrm{~s}$ structures had not been detected, leading to the speculation that magnetic splitting, instead of rotation, was responsible for the existence of the two "doublets". This is clearly not the case, as demonstrated by our CFHT observations.

Figure 6 contrasts the full frequency spectrum with the modeled frequency spectrum based on the extracted periods. On the lower part of the graph, we illustrate the point-by-point subtraction of the two curves. The smoothness of this curve is the last check that the obtained periods are quite consistent with the CFHT light curve of R548, and that no false detection has been introduced in the extraction process.

We will refer the reader to [5] for a detailed description of the methodology and the presentation of the results obtained from the asteroseismological optimization exercise carried out on the basis of the frequencies derived here for R548.

\section{References}

[1] Charpinet, S., Brassard, P., Fontaine, G., et al. 2009, American Institute of Physics Conference Series, 1170, 585

[2] Fontaine, G., \& Brassard, P. 2008, PASP, 120, 1043

[3] Bischoff-Kim, A., Montgomery, M.H., \& Winget, D.E. 2008, ApJ, 675, 1505

[4] Romero, A.D., Córsico, A.H., Althaus, L.G., et al. 2012, MNRAS, 420, 1462

[5] Giammichele, N., Fontaine, G., \& Brassard, P. 2012, in Proc. 18th European Workshop on White Dwarfs, ASP Conference Series, in press

[6] Montgomery, M.H., \& O'donoghue, D. 1999, Delta Scuti Star Newsletter, 13, 28 